\title{
BMJ Open Therapeutic management of complex anal fistulas by installing a nitinol closure clip: study protocol of a multicentric randomised controlled trial-FISCLOSE
}

\author{
Anne Dubois, ${ }^{1}$ Guillaume Carrier, ${ }^{1}$ Bruno Pereira, ${ }^{2}$ Brigitte Gillet, ${ }^{1}$ \\ Jean-Luc Faucheron, ${ }^{3}$ Denis Pezet, ${ }^{1,2,4,5}$ David Balayssac ${ }^{2,5,6}$
}

To cite: Dubois A, Carrier G, Pereira B, et al. Therapeutic management of complex anal fistulas by installing a nitinol closure clip: study protocol of a multicentric randomised controlled trial-FISCLOSE. BMJ Open 2015;5:e009884. doi:10.1136/bmjopen-2015009884

\section{- Prepublication history} and additional material is available. To view please visit the journal (http://dx.doi.org/ 10.1136/bmjopen-2015009884).

Received 1 September 2015 Revised 5 November 2015 Accepted 10 November 2015

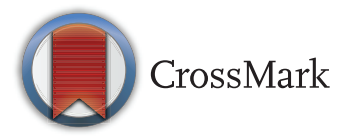

For numbered affiliations see end of article.

Correspondence to Dr Anne Dubois; a_dubois@chuclermontferrand.fr

\section{ABSTRACT}

Introduction: Complex anal fistulas are responsible for pain, faecal incontinence and impaired quality of life. The rectal mucosa advancement flap (RMAF) procedure to cover the internal opening of the fistula remains a strategy of choice. However, a new procedure for closing anal fistulas is now available with the use of a nitinol closure clip (OTSC Proctology, OVESCO), which should ensure a better healing rate. This procedure is currently becoming more widespread, though without robust scientific validation, and it is therefore essential to carry out a prospective evaluation in order to determine the efficacy and safety of this new medical device for complex anal fistulas.

Methods and analysis: The FISCLOSE trial is aimed at evaluating the efficacy and safety of a nitinol closure clip compared to the RMAF procedure for the management of complex anal fistulas. This trial is a prospective, randomised, controlled, single-blind, bicentre and interventional study. Patients $(n=46$ per group) will be randomly assigned for management with either a closure clip or RMAF. The main objectives are to improve the healing rate of the anal fistula, lessen the postoperative pain and faecal incontinency, enhance the quality of life, and lower the number of reinterventions and therapeutic management costs. The primary outcome is the proportion of patients with a healed fistula at 3 months. The secondary outcomes are anal fistula healing (6 and 12 months), proctological pain (visual analogue scale), the faecal incontinence score (Jorge and Wexner questionnaire), digestive disorders and quality of life (Gastrointestinal Quality of Life Index and Euroqol EQ5D-3 L) up to 1 year.

Ethics and dissemination: The study was approved by an independent medical ethics committee 1 (IRB00008526, CPP Sud-Est 6, Clermont-Ferrand, France) and registered by the competent French authority (ANSM, Saint Denis, France). The results will be disseminated in a peer-reviewed journal and presented at international congresses.

Trial registration number: NCT02336867; pre-result.

\section{INTRODUCTION}

Anal fistulas are the main aetiology of perianal abscesses and suppurations. They are common and generally associated with pain, faecal incontinence, impaired quality of life and work incapacity. The therapeutic management involved has a twofold objective: heal suppuration and preserve sphincter function. ${ }^{2}$ Each year, anal fistulas affect more than 1:10000 individuals, ${ }^{3}$ with a higher prevalence in men than in women (1.23 per 10000 men and 0.56 per 10000 women $)^{4}$ and an average age of 40 years. ${ }^{1}$

In about $80 \%$ of cases, anal fistulas are secondary to an infection of the Hermann and Desfosses anal glands (cryptogenic or cryptoglandular). This anal gland infection can lead to an abscess between the internal and external sphincters, which can also spread to other parts of the perianal region. The infection can follow many directions from this point in the intersphincteric space. The fistula is formed when the pus reaches the skin. The origin of anal fistulas is therefore always intraductal, cryptic, with a primary port at this level and usually a secondary port in the skin. Although the classification of anal fistulas is still under debate, they are usually divided into two groups. The first group contains what is known as 'simple' fistulas, which are intersphincteric or transsphincteric fistulas involving only the lower third of the sphincter complex. The second group contains what is known as 'complex' fistulas. This second type of fistula can be intersphincteric, trans-sphincteric or even suprasphincteric and extrasphincteric. ${ }^{135}$

For many years, fistulotomy was the treatment of choice, but this procedure was associated with a significant risk of faecal 
incontinence in about $30 \%$ of patients with the severity of faecal incontinence increasing with the complexity of the fistula. ${ }^{6-9}$ Other surgical treatments were developed, with more or less success, such as setons, fibrin glue, collagen plugs and the rectal mucosa advancement flap (RMAF) procedure to cover the internal opening of the fistula. The RMAF procedure has become a strategy of choice in the treatment of anal fistulas and particularly in the case of complex fistulas. ${ }^{10}{ }^{11}$ The success rate of this procedure has recently been determined at around $60 \%$. $^{41213}$

A new procedure for closing anal fistulas is currently being developed with the use of a nitinol closure clip (OTSC Proctology device) derived from the over-the-scope-clip (OTSC) (OVESCO endoscopy AG, Tübingen, Germany). This new procedure has been validated in a porcine model of an anal fistula, ensuring the safety of the device. ${ }^{14}$ The first case was published for a patient with a complex anal fistula (high transsphincteric).$^{15}$ After erosion of the fistula tract with a special brush, a nitinol clip (OTSC Proctology) was deposited on the internal opening of the fistula. Eight months after surgery, the fistula was healed and the clip was removed by cutting with a clip cutter. ${ }^{15}$ Thereafter, the OTSC closure clip was assessed in a prospective, non-randomised and non-blinded clinical trial. Ten patients were enrolled for the management of complex anal fistulas (nine trans-sphincteric and one suprasphincteric fistulas). After follow-up of 6 months, the healing rate was about $90 \% .^{16}$ Recently, a retrospective monocentre study enrolled 10 patients with refractory trans-sphincteric fistulas $(6 / 10$ perianal Crohn's disease). The healing rate was about $70 \%$ for a median follow-up time of 72 days (min-max: 31-109 days). ${ }^{17}$ The safety of the OTSC closure clip was good, since only 3 of the 20 patients included experienced slight anal discomfort. ${ }^{16} \quad{ }^{17}$ However, another recent retrospective study on 17 patients found a very low healing rate of $12 \%$ after a short follow-up (mean: 4 months). ${ }^{18}$ This study highlighted numerous complications with $65 \%$ of the patients experiencing pain or clip migration. However, it is noteworthy that this retrospective study conducted in six centres included a heterogeneous population of patients with high fistulas ( $71 \%$ with 4 rectovaginal fistulas), lower fistulas (29\% with 3 rectovaginal fistulas) and Crohn's fistulas (35\%). The study by Gautier $e t a l^{18}$ also has a high technical failure rate with $53 \%$ difficult procedures and $64.7 \%$ clip migrations. Moreover, other authors underlined that these patients do not represent the core of the indication spectrum for the OTSC closure clip, but, rather, is at the margin of what this device is indicated for. ${ }^{19}$

This procedure is currently becoming more widespread and dozens of patients have been treated with the nitinol closure clip, though without robust scientific validation of the process. To date, this innovative nitinol closure clip has not yet been assessed in a randomised controlled trial. It is therefore essential to carry out a prospective evaluation in order to determine the efficacy and safety of this new medical device in the case of complex anal fistulas.

\section{METHODS AND ANALYSIS \\ Study design}

The present study is a prospective, randomised, controlled, single-blind and bicentre, phase II/III trial that compares a nitinol closure clip versus the RMAF procedure to close complex anal fistulas.

\section{Study objectives}

In the experimental group (OTSC clip) compared with the control group (RMAF procedure), the main objective will be to demonstrate an improvement of the healing rate of the anal fistula 3 months after intervention. The other objectives will be to demonstrate an improvement in the healing rate, postoperative pain, faecal incontinency and quality of life, and fewer re-interventions and reduced therapeutic management costs.

\section{Inclusion and exclusion criteria}

To be included in the study, the patient must have a complex anal fistula (trans-sphincteric, suprasphincteric, extrasphincteric), drained and requiring a closing intervention. Patients must be naive to any surgical treatment for fistula closure, subscribe to the French national health insurance system and give their written consent.

Patients cannot be included in the study for one of the following criteria: $<18$ and $>80$ years of age, body mass index (BMI) $>35 \mathrm{~kg} / \mathrm{m}^{2}$, rectovaginal or rectourethral fistulas, distal trans-sphincteric and intersphecteric fistulas, Crohn's disease, infections (sepsis, tuberculosis or HIV infection), nickel allergy, cognitive disorders or major disability making it impossible to understand the study and sign the informed consent, breastfeeding or pregnancy. Patients suffering from Crohn's disease develop more anal fistulas and postsurgery complications. ${ }^{17}$ Finally, patients already included in another clinical trial not compliant with the criteria of the study or with legal incapacity (person deprived of liberty or subject to guardianship) cannot be included in this study.

Stopping guidelines will include withdrawal of patient consent, non-compliance by the patient, adverse event and by decision of the investigator. In case of withdrawal from the study, the patient will be followed and managed normally in the digestive surgery department.

The exclusion period during which the patient cannot participate in another clinical trial is 15 days before inclusion and 0 days after the end of the study.

\section{Interventions}

The abscess must be drained before the closure intervention. The abscess will be opened and drained with an internal seton, which must remain in place for 
6 weeks. Thereafter, and depending on the randomisation, the internal opening of the fistula will be closed using either the RMAF procedure (control group) or the nitinol closure clip (innovative device group).

The anaesthesia procedure will be identical to that of conventional proctological surgery, namely, general anaesthesia with or without pudendal block. During general anaesthesia, muscle relaxation (curare) is ensured to protect the sphincter specifically for the RMAF procedure, which requires the use of a spacer that is potentially harmful for the sphincter. If there are any particular problems, it will be possible to use a local anaesthetic.

\section{MRAF procedure}

Originally developed for rectovaginal fistulas, MRAF has been transposed to cryptoglandular fistulas. The principle is to close the internal opening by the interposition of a rectal mucosa wall flap and ensure the drying of the fistula. Analysis of the literature is difficult because most trials have mixed cryptoglandular and rectovaginal fistulas in addition to a variable proportion of Crohn's disease. The current healing rate is close to $60 \%$ and the risk of faecal incontinence remains about $0-52 \%$, depending on the study. ${ }^{4-8} 1213$

As there are many surgical variants of the RMAF procedure, investigators will agree on performing a harmonised procedure to ensure reproducible results. Briefly, the anal canal and internal opening of the fistula will be exposed with a bivalve spacer or a Lone Star spacer. The tract of the fistula will be prepared by first removing the seton, abrasion of the tract with a brush (OTSC fistula brush, OVESCO) and flushing the fistula tract with sterile saline solution. The flap will be prepared by an incision at the upper part for internal opening by electrocautery (one-third of the circumference and totoparietal plane). The mucosa and submucosa will be removed $(1-2 \mathrm{~mm})$ around the internal opening. The transmuscular opening will be closed with 1 or 2 sutures (3.0 or 4.0, PDS, Ethicon). Thereafter, a panparietal and vascularised flap will be dissected in order to lower the flap on the linea pectinea. Haemostasis must be performed correctly to limit haematomas. The flap is lowered and fixed with separate sutures on a single plane (3.0 or 4.0, PDS, Ethicon). The choice of a slowresorbing suture is motivated by the risk of late relapse after fast resorption of the suture. The external opening is left open to drain the flap and avoid the formation of a haematoma or secondary abscesses in the fistula tract. No transanal drainage will be performed. Finally, the watertightness of the closure will be tested by irrigation of sterile saline solution over the external fistula opening. In case of leakage, the flap will be reinforced with one or several elective sutures.

\section{Nitinol closure clip procedure}

The OTSC Proctology system is derived from the OTSC, Over-The-Scope Clip procedure used by gastrointestinal endoscopists to dry up digestive fistulas and close colonoscopic perforations. These procedures use the same medical device, which is a shape memory nitinol clip that provides dynamic closure of the fistula orifice. Compared to a suture, which is rather static, the nitinol closure clip provides dynamic compression of the opening.

The OTSC Proctology kit contains a disposable applicator loaded with a nitinol clip, a brush and a preset thread retriever. The estimated cost in France is currently $€ 690$ per procedure $(\$ 770$ ) (data from the laboratory).

The closure clip procedure begins with the same proctological control, under general anaesthesia, as the advancement flap procedure, using a bivalve spacer or a Lone Star spacer. The fistula tract must be drained with a loose seton for at least for 6 weeks before closure of the opening in order to obtain a non-suppurating tract without any residual abscess. Thereafter, the seton is removed, and the fistula tract is eroded with the brush (OTSC fistula brush, OVESCO) and flushed with sterile saline solution. The epithelium is removed circumferentially around the internal opening of the fistula to a depth of several millimetres to allow positioning the closure clip on the underlying muscular tissue in order to limit postoperative pain. To facilitate the closure clip placement procedure, two U-shaped sutures $(2.0,90 \mathrm{~cm}$, Vicryl, Ethicon) can be placed through the sphincter muscle centring the internal opening of the fistula (12$18 \mathrm{~h}$ and $15-21 \mathrm{~h}$ ). The ends of the threads are pulled through the working channel of the instrument by means of the thread retriever. With the two U-shaped sutures taut, the applicator is inserted in the anus, and applied above the fistula opening and parallel to the fistula tract. The closure clip is fitted on the fistula opening and the two U-shaped sutures are cut. The clip position is controlled by the closure of the internal opening of the fistula and clasps the muscular part without signs of local ischaemia. The watertightness of the closure will be tested by irrigation of sterile saline solution over the external fistula opening. In case of leakage, the clip will be removed and replaced by a new one until definitive closure. If necessary, the clip can be removed with a specific clip cutter.

\section{Study outcomes}

The primary outcome is the rate of patients with a healed anal fistula 3 months after surgery. The diagnosis will be determined by the lack of leakage alleged by the patient for at least 1 month and confirmed by clinical examination (table 1).

The secondary end points are anal fistula healing (6 months and 1 year after surgery), proctological pain assessed with a visual analogue scale (VAS) (at days: 0,1 , $2,3,15,30,60,90,180$ and 365), the faecal incontinence score assessed with the Jorge and Wexner questionnaire $^{20}$ (at days: $0,15,30,60,90,180$ and 365), digestive disorders, behaviour of the clip (spontaneous detachment, ingrowth, migration) and quality of life assessed 
Table 1 Time schedule of enrolment, intervention and assessments

\begin{tabular}{|c|c|c|c|c|c|c|c|c|c|}
\hline $\begin{array}{l}\text { Days } \\
\text { Visits }\end{array}$ & $\begin{array}{l}\text { Inclusion } \\
1\end{array}$ & $\begin{array}{l}0 \\
2\end{array}$ & $1-3$ & $\begin{array}{l}15 \\
3\end{array}$ & $\begin{array}{l}30 \\
4\end{array}$ & $\begin{array}{l}60 \\
5\end{array}$ & $\begin{array}{l}90 \\
6\end{array}$ & $\begin{array}{l}180 \\
7\end{array}$ & $\begin{array}{l}365 \\
8\end{array}$ \\
\hline $\begin{array}{l}\text { Patients characteristics } \\
\text { Medical history }\end{array}$ & $x$ & & & & & & & & \\
\hline Surgery & & $x$ & & & & & & & \\
\hline Physical examination & $X$ & $x$ & & $x$ & $x$ & $\mathrm{X}$ & $\mathrm{X}$ & $x$ & $x$ \\
\hline Proctological examination & $x$ & $\mathrm{X}$ & & $x$ & $x$ & $\mathrm{X}$ & $x$ & $x$ & $x$ \\
\hline Adverse event monitoring & & $x$ & $x$ & $\mathrm{X}$ & $\mathrm{X}$ & $\mathrm{X}$ & $\mathrm{X}$ & $x$ & $x$ \\
\hline Clip behaviour & & $x$ & $\mathrm{X}$ & $x$ & $\mathrm{X}$ & $x$ & $x$ & $x$ & $\mathrm{X}$ \\
\hline Analgesic drug use & $\mathrm{X}$ & $x$ & $\mathrm{X}$ & $\mathrm{X}$ & $x$ & $X$ & $x$ & $x$ & $x$ \\
\hline Fistula healing & & & & & & & $x$ & $x$ & $x$ \\
\hline Proctological pain (VAS) & & $\mathrm{X}$ & $\mathrm{X}$ & $\mathrm{X}$ & $\mathrm{X}$ & $\mathrm{X}$ & $\mathrm{X}$ & $\mathrm{X}$ & $\mathrm{X}$ \\
\hline Jorge and Wexner, GIQLI, EQ5D questionnaires & & $\mathrm{X}$ & & $\mathrm{X}$ & $\mathrm{X}$ & $\mathrm{X}$ & $\mathrm{X}$ & $\mathrm{X}$ & $\mathrm{X}$ \\
\hline
\end{tabular}

with the Gastrointestinal Quality of Life Index $\left(\right.$ GIQLI) ${ }^{21}$ (at days: 0, 15, 30, 60, 90, 180 and 365), and the quality of life assessed with the Euroqol EQ5D-3L Questionnaire (at days: 0, 30, 90 and 365) ${ }^{22}$ (table 1). Complex anal fistulas and faecal incontinence alter patient's quality of life. ${ }^{9}$

\section{Methodology and study design}

The trial will be performed at two centres. The patients will be recruited, treated and followed-up at the digestive surgery department of the University Hospital of Clermont-Ferrand (France) and at the digestive surgery department of the University Hospital of Grenoble (France).

After controlling the inclusion and exclusion criteria, the FISCLOSE trial will be proposed by surgeons (AD, DP, JLF) to patients suffering from a complex anal fistula and requiring a closure intervention. The patients will be informed of the trial protocol and, on acceptance, will be randomised in the OTSC clip group or the control group. Randomisation will be carried out using a dedicated centralised telephone system, accessible round the clock, and using a computer-generated allocation sequence. The randomisation sequence will be generated by a biostatistician (BP) using random blocks and stratification as a function of the surgeons.

The trial will be single blinded because of the surgery techniques employed. The patient will not be informed of the randomisation arm throughout the trial, except in the case of adverse reaction.

Surgery will be performed by three surgeons and the possible 'surgeon effect' will be assessed in the statistical analysis. However, with only two inclusion centres, the surgical procedures will be easy to harmonise between surgeons and centres. The three surgeons have validated and harmonised the OTSC clip and RMAF procedures.

\section{Statistical considerations}

Estimation sample size

According to our previous works and the literature, ${ }^{16}$ the success rate (proportion of patients without healed anal fistula, 3 months after surgery) with the expected OTSC Proctology technique should be equal to $90 \%$. Therefore, considering a $60 \%$ success rate of the advancement flap procedure, ${ }^{4} \mathrm{n}=42$ patients per group would be required to highlight a significant difference between the two groups for a two-sided type-I error of $5 \%$ and $90 \%$ statistical power. Finally, a total of 92 patients will be considered. An interim analysis is planned after enrolment of the first 46 patients $(n=23 \times 2)$ using the Obrien-Fleming method (East software, Cytel Inc, Cambridge, Massachusetts, USA).

\section{Statistical analysis}

Statistical analysis will be conducted on intention to treat using Stata software, V.13 (StataCorp, College Station, Texas, USA). A two-sided $p$ value of less than 0.05 will be considered to indicate statistical significance (except interim analysis). Baseline characteristics will be presented for each randomised group as the mean \pm standard-deviation or the median (IQR) according to the statistical distribution for continuous data, and as the number of patients and associated percentages for categorical parameters.

Comparisons between independent groups will be analysed using the $\chi^{2}$ or Fisher's exact test for categorical variables (notably primary outcome, proportion of patients with a healed anal fistula 3 months after surgery) and the Student t test or Mann-Whitney's test for quantitative parameters, with normality verified by the Shapiro-Wilk test and homoscedasticity by the Fisher-Snedecor test.

Concerning the primary analysis, the univariate analysis will be completed in the second step by multivariate analysis performed using a generalised linear model. Covariates used for adjustment will be fixed according to (1) univariate results, (2) stratification factors and (3) clinical relevance such as: BMI, diabetes, tobacco and drugs (anticoagulant, platelet antiaggregant, antiinflammatory, immunosuppressant). The possible surgeon effect will be studied through a random effect model. Results will be expressed as relative-risks and 
95\% CIs. Regarding the analysis of repeated measures (anal fistula healing, proctological pain assessed with a VAS, faecal incontinence score assessed with the Jorge and Wexner questionnaire, digestive disorders and quality of life assessed with GIQLI, quality of life assessed with the Euroqol EQ5D-3L), random-effect models (linear or generalised linear according to the statistical distribution of dependent variables) will be considered, as usually proposed, to study the fixed effects group, time points and interaction 'group $\mathrm{x}$ time', taking into account between and within-subject variability.

Although the amount of missing data should be limited by the fact that the monitoring of patients will be the standard therapeutic management of this disease, the estimation method developed by Verbeke and Molenberghs will be proposed to assess possible problems caused by missing data. ${ }^{23}$

\section{ETHICS AND DISSEMINATION Approval}

In accordance with the Declaration of Helsinki and French law on clinical trials, the study was presented to an independent medical ethics committee, the Comité de Protection des Personnes Sud Est 6 (IRB00008526, Clermont-Ferrand, France). The approval of the medical ethics committee was obtained on 4 April 2014. The protocol was declared to the competent French authority (Agence Nationale de Sécurité du Médicament et des produits de santé, Saint Denis, France) and registered under number 2014-A00441-46. Authorisation was obtained on 27 May 2014.

Any substantial change in the protocol and any change in the informed consent form will be presented to the independent medical ethics committee. The independent medical ethics committee and the competent French authority will be informed of the end of the study. In accordance with the independent medical ethics committee (Comité de Protection des Personnes Sud-Est 6, Clermont-Ferrand, France), no safety and data monitoring committee has been set up, considering the low risk of the intervention.

The study is currently registered on the clinical trials website under the following number: NCT02336867. The protocol has been in its fourth version since 5 December 2014.

\section{Patient informed consent}

According to French law on clinical trials, written informed consent must be obtained from patients prior to their participation in the study. Patients will voluntarily confirm their willingness to participate in the study after having been informed (in writing and verbally) by oncologists of all the aspects of the study relevant to their decision to participate. They will be informed about requirements concerning data protection and have to agree to direct access to their individual data. The patients will be informed that they are free to withdraw from the study at any time at their own discretion without necessarily giving reasons (see online supplementary files).

\section{Data collection and quality management}

Clinical research technicians are dedicated to data acquisition, coding, security and storage. Each included patient and study datum are anonymised. Study data are collected and managed using REDCap electronic data capture tools hosted at the University Hospital of Clermont-Ferrand. ${ }^{24}$ Research Electronic Data Capture (REDCap) is a secure, web-based application designed to support data capture for research studies, providing: (1) an intuitive interface for validated data entry; (2) audit trails for tracking data handling and export procedures; (3) automated export procedures for seamless data downloads to common statistical packages; and (4) procedures for importing data from external sources.

A clinical research assistant will be commissioned by the sponsor (University Hospital of Clermont-Ferrand) in order to monitor the progress of the study in accordance with the Standard Operating Procedures implemented at the University Hospital of Clermont-Ferrand, in accordance with the Good Clinical Practice and current French laws.

\section{Access to data and dissemination of results}

The data set will be the property of the sponsor (CHU Clermont-Ferrand). However, the principal investigator $(\mathrm{AD})$ and the project manager (DB) will have full access to the final data set. The results will be communicated in a peer-reviewed journal, presented at international congresses and completed online on ClinicalTrials.gov.

\section{DISCUSSION}

There is no consensus on the surgical treatment of anal fistulas at present. ${ }^{25}$ Management of complex anal fistulas remains a tremendous challenge because of the high level of recurrence and complications, depending of the type of fistula and choice of closure procedure. ${ }^{9} 17$ The initial results for the OTSC nitinol closure clip are very encouraging. ${ }^{16}{ }^{17}$ The OTSC clip combines a simple procedure and a sphincter-preserving approach that, for the moment, have demonstrated a very good healing rate with acceptable safety. The FISCLOSE study presented here should validate these preliminary results on the basis of a bicentre, randomised and controlled trial.

Author affiliations

${ }^{1} \mathrm{CHU}$ Clermont-Ferrand, service de Chirurgie Digestive, Clermont-Ferrand, France

${ }^{2} \mathrm{CHU}$ Clermont-Ferrand, Délégation à la Recherche Clinique et à l'Innovation, Clermont-Ferrand, France

${ }^{3} \mathrm{CHU}$ Grenoble, service de Chirurgie Digestive, Grenoble, France

${ }^{4}$ INSERM, U1071, Clermont-Ferrand, France 
${ }^{5}$ Université d'Auvergne, Clermont-Ferrand, France

${ }^{6}$ INSERM, U1107 NEURO-DOL, Clermont-Ferrand, France

Contributors AD, GC, DB, J-LF and DP led the conceptualisation, design and implementation of this research protocol. BP led the development of the statistical analysis plan. BG participated in the design of the protocol for interventions and assessments. All the authors have read and approved the final manuscript.

Funding The nitinol closure clips (OTSC Proctology) and the fistula Brush PR are generously provided by OVESCO laboratory (French provider: Life Partners). This study has also received a grant from $\mathrm{CHU}$ Clermont-Ferrand (AOI 2015).

Competing interests None declared.

Ethics approval IRB00008526 and ANSM.

Provenance and peer review Not commissioned; externally peer reviewed.

Data sharing statement The data will be stored by the CHU Clermont-Ferrand (France) and will be available on request at: $\mathrm{CHU}$-Clermont-Ferrand Délégation à la Recherche Clinique et à l'Innovation 58 rue Montalembert 63000 Clermont-Ferrand, France-Phone: +33 473751028 dbalayssac@chu-clermontferrand.fr.

Open Access This is an Open Access article distributed in accordance with the Creative Commons Attribution Non Commercial (CC BY-NC 4.0) license, which permits others to distribute, remix, adapt, build upon this work noncommercially, and license their derivative works on different terms, provided the original work is properly cited and the use is non-commercial. See: http:// creativecommons.org/licenses/by-nc/4.0/

\section{REFERENCES}

1. Simpson JA, Banerjea A, Scholefield JH. Management of anal fistula. BMJ 2012;345:e6705

2. Lindsey I, Smilgin-Humphreys MM, Cunningham $\mathrm{C}$, et al. A randomized, controlled trial of fibrin glue vs. conventional treatment for anal fistula. Dis Colon Rectum 2002;45:1608-15.

3. Tabry H, Farrands PA. Update on anal fistulae: surgical perspectives for the gastroenterologist. Can J Gastroenterol 2011;25:675-80.

4. Leng $Q$, Jin $H-Y$. Anal fistula plug vs mucosa advancement flap in complex fistula-in-ano: a meta-analysis. World J Gastrointest Surg 2012;4:256-61.

5. Parks AG, Gordon PH, Hardcastle JD. A classification of fistula-in-ano. Br J Surg 1976;63:1-12

6. Toyonaga T, Matsushima M, Kiriu T, et al. Factors affecting continence after fistulotomy for intersphincteric fistula-in-ano. Int $J$ Colorectal Dis 2007;22:1071-5.

7. Tozer $\mathrm{P}$, Sala S, Cianci V, et al. Fistulotomy in the tertiary setting can achieve high rates of fistula cure with an acceptable risk of deterioration in continence. J Gastrointest Surg 2013;17:1960-5.
8. Arroyo A, Pérez-Legaz J, Moya P, et al. Fistulotomy and sphincter reconstruction in the treatment of complex fistula-in-ano: long-term clinical and manometric results. Ann Surg 2012;255: 935-9.

9. Visscher AP, Schuur D, Roos R, et al. Long-term follow-up after surgery for simple and complex cryptoglandular fistulas: feca incontinence and impact on quality of life. Dis Colon Rectum 2015;58:533-9.

10. A ba-bai-ke-re M-M-T-J, Wen H, Huang H-G, et al. Randomized controlled trial of minimally invasive surgery using acellular dermal matrix for complex anorectal fistula. World $J$ Gastroenterol 2010;16:3279-86

11. van der Hagen SJ, Baeten CG, Soeters PB, et al. Long-term outcome following mucosal advancement flap for high perianal fistulas and fistulotomy for low perianal fistulas: recurrent periana fistulas: failure of treatment or recurrent patient disease? Int $J$ Colorectal Dis 2006;21:784-90.

12. Soltani A, Kaiser AM. Endorectal advancement flap for cryptoglandular or Crohn's fistula-in-ano. Dis Colon Rectum 2010;53:486-95.

13. Mitalas LE, Dwarkasing RS, Verhaaren R, et al. Is the outcome of transanal advancement flap repair affected by the complexity of high transsphincteric fistulas? Dis Colon Rectum 2011;54:857-62.

14. Prosst RL, Herold A, Joos AK, et al. The anal fistula claw: the OTSC clip for anal fistula closure. Color Dis 2012;14:1112-17.

15. Prosst RL, Ehni W. The OTSC $®$ Proctology clip system for anorectal fistula closure: the 'anal fistula claw': case report. Minim Invasive Ther Allied Technol 2012;21:307-12.

16. Prosst RL, Ehni W, Joos AK. The OTSC® Proctology clip system for anal fistula closure: first prospective clinical data. Minim Invasive Ther Allied Technol 2013;22:255-9.

17. Mennigen R, Laukötter M, Senninger N, et al. The OTSC(®) proctology clip system for the closure of refractory anal fistulas. Tech Coloproctology 2015;19:241-6.

18. Gautier M, Godeberge P, Ganansia R, et al. Easy clip to treat anal fistula tracts: a word of caution. Int J Colorectal Dis 2015;30:621-4.

19. Schurr MO, Prosst RL. Comment on: easy clip to treat anal fistula tracts: a word of caution by M. Gautier et al. Int J Colorectal Dis 2015. Published Online First: 17 April 2015.

20. Jorge JM, Wexner SD. Etiology and management of fecal incontinence. Dis Colon Rectum 1993;36:77-97.

21. Slim K, Bousquet J, Kwiatkowski F, et al. [First validation of the French version of the Gastrointestinal Quality of Life Index (GIQLI)]. Gastroentérol Clin Biol 1999;23:25-31.

22. Chevalier J, de Pouvourville G. Valuing EQ-5D using time trade-off in France. Eur J Heal Econ 2013;14:57-66.

23. Verbeke G, Molenberghs G. Linear mixed models for longitudinal data. New York: Springer, 2009.

24. Harris PA, Taylor R, Thielke R, et al. Research electronic data capture (REDCap) - a metadata-driven methodology and workflow process for providing translational research informatics support. $J$ Biomed Inform 2009;42:377-81.

25. Ratto C, Litta F, Donisi L, et al. Fistulotomy or fistulectomy and primary sphincteroplasty for anal fistula (FIPS): a systematic review. Tech Coloproctology 2015;19:391-400. 